\title{
Extensive Gastroesophageal Varices in a Non-Cirrhotic Female with Left Isomerism: A Case Report and Literature Review
}

\author{
Maryam Rahmani, ${ }^{1}$ Faeze Salahshour, ${ }^{1,}$ Reza Taslimi, ${ }^{2}$ Ali Borhani, ${ }^{1}$ and Sina Kianoush ${ }^{3}$ \\ ${ }^{1}$ Advanced Diagnostic and Interventional Radiology Research Center, Tehran University of Medical Sciences, Tehran, Iran \\ ${ }^{2}$ Department of Internal Medicine, Imam Khomeini Hospital, Tehran University of Medical Sciences, Tehran, Iran \\ ${ }^{3}$ The Johns Hopkins Ciccarone Center for the Prevention of Heart Disease, Baltimore, Maryland, USA \\ "Corresponding author: Faeze Salahshour, Advanced Diagnostic and Interventional Radiology Research Center, Tehran University of Medical sciences, Tehran, Iran. Tel: \\ +98-9127953120, E-mail: Salahshour_faeze@yahoo.com
}

Received 2015 November 24; Revised 2016 February 15; Accepted 2016 March 14

\begin{abstract}
Heterotaxy syndrome with polysplenia is a congenital abnormality with malposition of the visceral organs and multiple associated abnormalities. The majority of affected individuals die during childhood due to severe cardiovascular anomalies. However, affected individuals who reach adulthood are usually asymptomatic. Anomalies of the abdominal venous system, including interruption of the inferior vena cava with azygos or hemiazygos continuation, congenital absence of the portal vein with a portosystemic shunt, preduodenal portal vein, and abnormalities of renal veins are frequent findings. Heterotaxy syndrome usually has no clinical significance except in the setting of planned vascular intervention or surgery. We are reporting an extremely rare case of heterotaxy syndrome (polysplenia) that presented with massive variceal hemorrhage due to associated venous malformation and systemic to portal venous system collaterals.
\end{abstract}

Keywords: Left Isomerism, Heterotaxy, Interruption of Inferior Vena Cava, Azygos Continuation, Gastroesophageal Varices, Congenital Absence of Portal Vein, Congenital Venous Stenosis

\section{Introduction}

Heterotaxy syndrome with polysplenia is a congenital abnormality with malposition of visceral organs and multiple associated anomalies such as cardiovascular malformations, bowel malrotation, short pancreas, biliary atresia, and preduodenal portal vein. The majority of patients die during childhood, mainly due to severe cardiovascular anomalies. However, about 5\% -10\% of affected individuals with no major cardiac anomaly reach adulthood and are nearly asymptomatic (1).

We are reporting an exceedingly rare case of heterotaxy syndrome (polysplenia) who presented at the age of 29 with massive upper gastrointestinal bleeding and extensive gastroesophageal varices due to associated venous malformation and systemic to portal venous system collateral vessels. Initially, the patient was misdiagnosed as a case of cirrhosis and underwent many avoidable medical interventions and ultimately died from massive uncontrollable upper gastrointestinal bleeding.

\section{Case Presentation}

The patient was a 29-year-old non-smoker nonalcoholic rural woman, who presented with massive upper gastrointestinal (GI) bleeding. She was admitted to a local general hospital with the impression of nonsteroidal anti-inflammatory drug (NSAID)-related gastritis or peptic ulcer disease. Upper endoscopic examination revealed multiple large varicose veins with active bleeding along the entire esophagus and stomach from the fundus to the antrum. No ulcers were found and rapid urease test for Helicobacter pylori was negative. Sclerotherapy and band ligation of the varices were performed during the first admission. Varices were attributed to portal hypertension and the patient underwent work-up for chronic liver disease and splenoportal vein thrombosis.

Apart from a remote episode of upper GI bleeding, which had not been evaluated, she had an unremarkable past medical history and no stigmata of chronic liver disease on physical examination. Liver function tests (bilirubin, aspartate aminotransferase, alanine aminotransferase), alkaline phosphatase, total protein, albumin, ceruloplasmin, markers of autoimmune hepatitis (anti smooth muscle antibody, ANA, IgG, IgM, IgA), and tests of blood coagulation (prothrombin time, international normalization ratio, protein $\mathrm{C}$, protein $\mathrm{S}$, anti-thrombin III, anti-lupus antibody, and platelet count) were within the normal limits.

On ultrasound exam, the organ in the right upper quadrant (RUQ) of the abdomen was misdiagnosed as the liver; consequently transient elasotography (FibroScan) 
and a blind biopsy were performed in order to confirm cirrhosis as the causing factor of variceal hemorrhage. FibroScan findings were suggestive of cirrhosis (F4). However, histopathological evaluation of biopsy specimen showed spleen tissue.

A magnetic resonance venography (MRV) was done in our center with the suspicion of splenic vein thrombosis. Right-sided large main spleen with adjacent smaller spleens (polysplenia), right sided stomach, midline normal liver, abnormal direction of the gallbladder, interrupted inferior vena cava (IVC) as the absence of intrahepatic and suprarenal portion of IVC, and enlarged azygos and hemiazygos systems suggesting azygos continuation were evident in MRV. On the other hand, the caudal portion of the azygos vein which should be anastomosed with the cranial portion of interrupted IVC was markedly narrowed and hypoplastic. Instead, there was an abnormal vein arising from the right side of the most cranial portion of IVC, just above the right renal vein coursing cephalad and ending in a network of collateral vessels around the stomach (Figure 1).

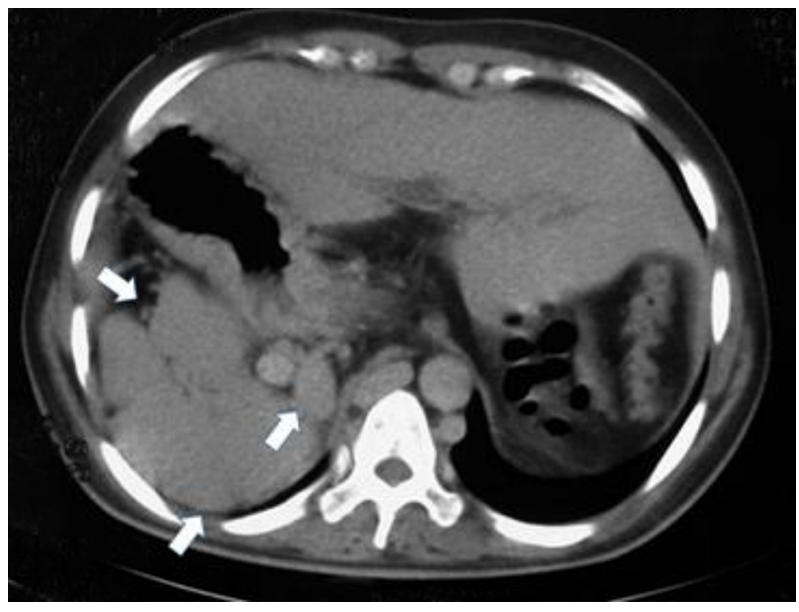

Figure 1. A 29-year-old woman presented with variceal massive upper gastrointestinal bleeding diagnosed as heterotaxy syndrome. Axial CT-scan image shows two almost normal-sized spleens and a smaller one in the right upper quadrant of the abdomen (white arrows)

Collateral veins were also present in perisplenic and subphrenic regions. No distinct splenic and portal veins were identified and collateral veins were recognized in their usual location. Apparent SMV was present, but this vein was also draining to the aforementioned perigastric venous collaterals (Figure 2). Prominent variceal vessels were observed around the esophagus, stomach, and in particular, esophagogastric junction.

Mild dilation of the intrahepatic bile ducts, bowel malrotation, circumaortic left renal vein, and congested pelvic veins were other significant findings. In the chest computerized tomography (CT) scan, superior vena cava (SVC) seemed unremarkable without evidence of obstruction, dilated azygos vein was draining to SVC and some prominent vessels were observed in the lower zone of the right lung.

Further diagnostic and therapeutic procedures, including catheter portovenography, echocardiography (for the detection of probable cardiac anomaly), and targeted Doppler ultrasound were planned but refused by the patient. One month after discharge, the patient died from massive uncontrollable upper GI bleeding.

\section{Discussion}

Based on MRV images, a diagnosis of congenital absence of the portal vein and extrahepatic portosystemic shunt was made. Congenital extrahepatic portosystemic shunts have two main types $(2,3)$. In type 1 , which is associated with polysplenia syndrome, there is no distinct portal vein, and splenic and mesenteric veins (separately (1a) or after forming a common trunk (1b)) drain to a systemic vein, which could be IVC, renal vein, iliac vein, azygos vein or even the right atrium. In type 2 , which less commonly has associated abnormalities, intrahepatic portal vein branches exist, and a side to side shunt connects portal vein with a systemic vein $(4,5)$.

Patients with type 1 congenital extrahepatic portosystemic shunt usually present with signs of encephalopathy, hepatopulmonary syndrome or benign liver masses, but esophageal varices are not a common feature of this entity and presence of varices recommends other causes of extrahepatic portal vein obstruction $(2,5)$.

In case of our patient, no distinct portal vein was seen, and extensive varices existed from the proximal esophagus to the distal stomach, which could be resulted from anomaly of the systemic veins. Azygos continuation of IVC was incomplete and the anastomosis of azygos vein to the IVC was severely hypoplastic. We assume that the above-mentioned abnormal vein from the superior part of IVC contributed to drainage of the interrupted IVC, and bypassed hypoplastic segment of the azygos vein. This vein drained systemic venous blood through gastric veins which resulted in congestion of gastric veins and formation of extensive varices, which then drained to azygos vein and therefore bypassed hypoplastic segment. This draining vein could be the inferior phrenic vein, which establishes extensive anastomoses with splenic and gastroepiploic veins. Alternatively, the vein could be a congenital extrahepatic portosystemic shunt that due to malformation of the caval system and interrupted drainage 

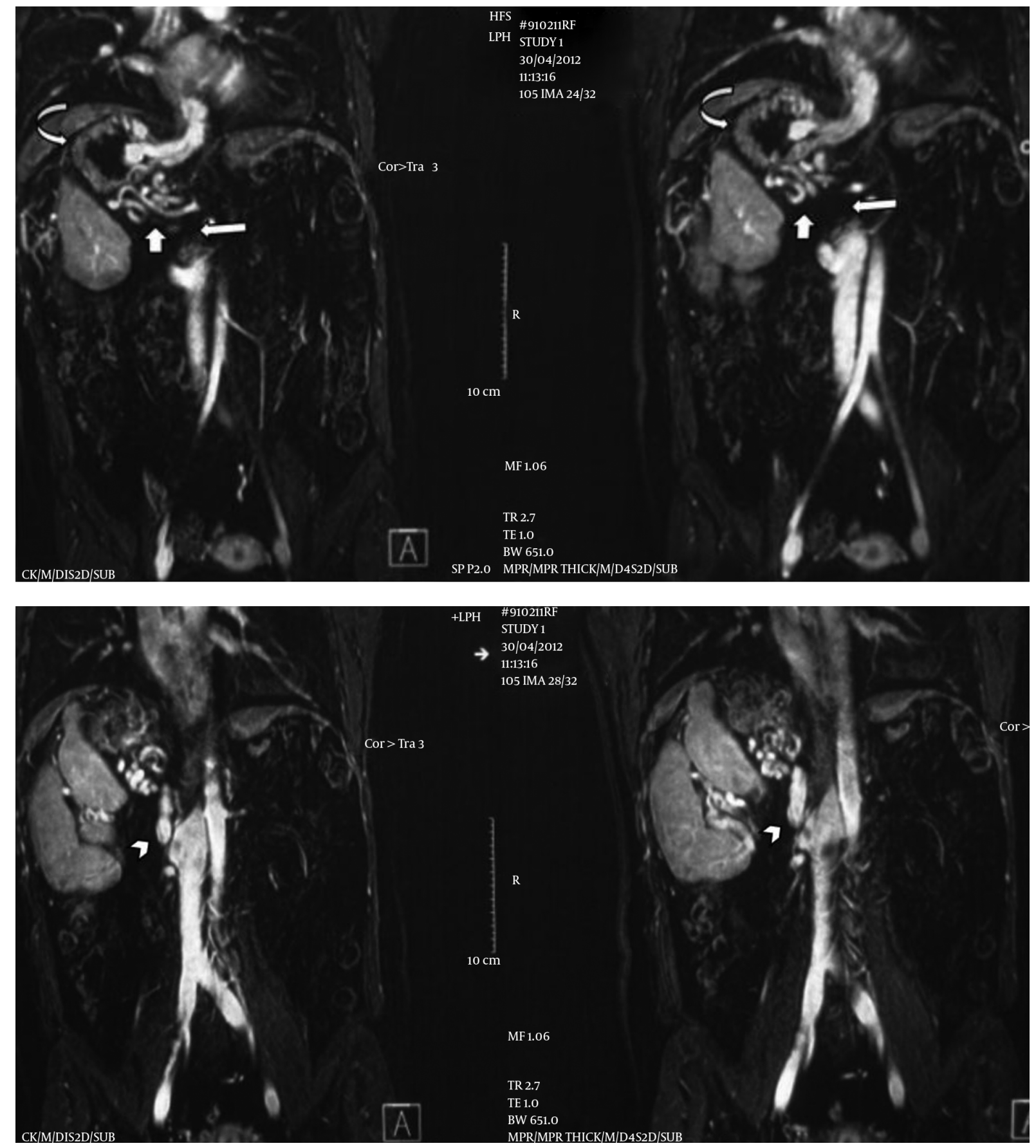

Figure 2. Coronal magnetic resonance venography images show a right-sided large main spleen with adjacent smaller spleens (polysplenia), right sided stomach, absence of suprarenal and intrahepatic segments of inferior vena cava (IVC) (long arrow) and azygos continuation. An abnormal vein (arrowhead) connects the infrarenal IVC to a network of varicose veins (short arrow) around the right-sided stomach (curved arrow).

of IVC acts inversely to drain systemic blood through gastrosplenic veins. As a result of absence of the intrahepatic portal system, drainage of the splanchnic blood was also through the perigastric collateral plexus and gastroesophageal veins. The prominent vessel in the lower lung was probably a prominent bronchial vessel filled by subdi- 
aphragmatic collaterals.

Anomalies of IVC are rare and found in less than $1 \%$ of the population $(6,7)$. In left isomerism, interruption of IVC is the second most consistent finding after multiple spleens (1).

Congenital interruption of IVC most commonly involves hepatic and suprarenal segments and agenesis of the infrarenal segment is less common (6). In this anomaly, which is usually asymptomatic, continuation of IVC most commonly establishes via the azygos or hemiazygos system, but other rare routes are also reported in the literature such as portal or hepatic veins (8-12). This anomaly is usually asymptomatic (13) and its clinical significance is mainly in the setting of cardiac catheterization and surgical explorations, in which unawareness regarding this anomaly could result in morbidity or mortality $(11,14,15)$. If a well-developed collateral pathway for continuation of IVC is not present, this anomaly could be symptomatic (6, 7). In a recent study conducted by Koc et al., $50 \%$ of the patients with interruption of IVC were symptomatic with deep vein thrombosis (DVT), leg pain and extremity varices and even one of the patients presented with hematochezia $(6,7)$. Several cases of low back pain and radiculopathy were attributed to IVC obstruction $(16,17)$ and even a rare case of painless hematuria that was caused by chronic post thrombotic obstruction of IVC (18) has been reported in the literature.

Generally, among patients with interrupted IVC or other causes of IVC obstruction, the symptoms result from blood stasis (recurrent DVT, leg pain, and extremity varicose) or formation of compensatory collateral vessels.

Common collateral pathways in patients with obstruction of SVC or IVC include cavocaval collaterals that direct blood through each other to the right atrium. These collaterals can be put into three groups: deep, superficial, and intermediate collaterals (19-21).

Superficial collaterals course in the abdominal wall and include two main pathways:

1, Proximal subclavian- internal/external mammarysuperior epigastric-inferior epigastric-external iliac; 2 , axillary vein-lateral thoracic vein-superficial epigastric/superficial circumflex iliac-common femoral vein; which connect tributaries of superior caval and inferior caval systems. Deep collaterals mainly consist of azygos and hemiazygos systems that caudally connect to common iliac veins via ascending lumbar veins, and cranially drain to SVC. Unless poorly developed, the azygos system is a predominant pathway in most cases (21). Deep collaterals also include the paraspinal and intra/extravertebral venous plexus $(19,21)$. Intermediate collaterals comprise the left gonadal vein and periureteric venous plexus, which develop in infrarenal obstruction of IVC and direct blood from the pelvis and lower extremity through renal veins to the suprarenal IVC or the hemiazygos system $(19,21)$. The aforementioned periureteric collaterals could explain gross hematuria that was reported as a complication of chronic IVC thrombosis. Other collateral pathways include cavopulmonary, cavoportal and intrahepatic collaterals (21). Cavopulmonary collaterals appear in SVC obstruction and consist of peribronchial venous plexus and collateral veins in pleural adhesions (21). The intrahepatic pathway forms in obstruction of upper IVC and develops mostly between hepatic veins to bypass the occluded hepatic segment of IVC (19). Small portal branches may also contribute to this pathway.

Although esophageal varices are a known complication of SVC obstruction, and few cases of duodenal and hemorrhoid varices are also reported as complication of segmental IVC agenesis or chronic thrombosis of IVC (2225), through our search in the literature, we did not find a case of extensive gastroesophageal varices as a complication of chronic obstruction or interruption of IVC. This case is a typical case of left isomerism, polysplenia, congenital absence of portal vein, interruption of IVC, incomplete azygos continuation with severe hypoplasia of anastomotic segment, and an abnormal vein that bypasses the hypoplastic segment of the azygos vein and drains systemic blood to gastric veins with resultant extensive gastroesophageal varices. Due to the absence of intrahepatic portal vein, splanchnic blood also drains via perigastric collateral vessels and gastroesophageal varices. Although most patients with left isomerism who reach adulthood are asymptomatic and this anomaly usually is found incidentally, our 29-year-old patient presented with upper gastrointestinal bleeding and eventually died from uncontrollable hemorrhage resulted from this complex venous anomaly.

We reported a rare case of heterotaxy syndrome (polysplenia) who presented with massive upper gastrointestinal hemorrhage due to complex venous anomaly. The patient was initially misdiagnosed as cirrhosis and underwent many unnecessary interventions and ultimately died from massive uncontrollable upper GI hemorrhage. Conclusively complex malformations of systemic veins should be kept in mind as a cause of gastrointestinal hemorrhage, particularly in polysplenia syndrome that is commonly associated with these anomalies.

\section{Footnotes}

Authors' Contributions: Study concept and design, Rahmani, Salahshour, and Taslimi; drafting of the manuscript, Salahshour; critical revision of the manuscript for important intellectual content, Borhani, and Kianoush. 
Financial Disclosure: There is nothing to disclose. Funding/Support: None declared.

\section{References}

1. Gayer G, Apter S, Jonas T, Amitai M, Zissin R, Sella T, et al. Polysplenia syndrome detected in adulthood: report of eight cases and review of the literature. Abdom Imaging. 1999;24(2):178-84. [PubMed: 10024407]

2. Murray CP, Yoo SJ, Babyn PS. Congenital extrahepatic portosystemic shunts. Pediatr Radiol. 2003;33(9):614-20. doi: 10.1007/s00247-003 1002-x. [PubMed: 12879313].

3. Niwa T, Aida N, Tachibana K, Shinkai M, Ohhama Y, Fujita K, et al. Congenital absence of the portal vein: clinical and radiologic findings. $J$ Comput Assist Tomogr. 2002;26(5):681-6. [PubMed: 12439298].

4. Kobayashi N, Niwa T, Kirikoshi H, Fujita K, Yoneda M, Saito S, et al. Clinical classification of congenital extrahepatic portosystemic shunts. Hepatol Res. 2010;40(6):585-93. doi: 10.1111/j.1872-034X.2010.00667.x. [PubMed: 20618456].

5. Alonso-Gamarra E, Parron M, Perez A, Prieto C, Hierro L, LopezSantamaria M. Clinical and radiologic manifestations of congenital extrahepatic portosystemic shunts: a comprehensive review. Radiographics. 2011;31(3):707-22. doi: 10.1148/rg.313105070. [PubMed: 21571652].

6. Koc Z, Oguzkurt L. Interruption or congenital stenosis of the inferior vena cava: prevalence, imaging, and clinical findings. Eur J Radiol. 2007;62(2):257-66. doi: 10.1016/j.ejrad.2006.11.028. [PubMed: 17161574].

7. Koc Z, Ulusan S, Oguzkurt L, Serin E. Symptomatic interrupted inferior vena cava: report of a case presenting with haematochezia. BrJ Radiol. 2007;80(954):e122-4. doi: 10.1259/bjr/31792102. [PubMed: 17684073].

8. Slosman F, Schmid MR, Pfammatter T. Agenesis of the hepatic segment of the inferior vena cava with portal continuation. AJR Am J Roentgenol. 2001;177(1):120-2. doi: 10.2214/ajr.177.1.1770120. [PubMed: 11418410].

9. Chevallier P, Peten EP, Marcy PY, Fabiani P, Diaine B, Padovani B. Inferior vena cava hypoplasia with intrahepatic venous continuation: sonographic, angiographic and MR features including MR angiography. Clin Imaging. 1999;23(2):99-102. [PubMed: 10416085].

10. Bercoff E, Colin R, Benozio M, Janvresse A, Berland J, Fillastre JP. Infrahepatic interruption of the inferior vena cava with portal continuation. Radiology. 1985;154(3):771-2. doi: 10.1148/radiology.154.3.3881797. [PubMed: 3881797].

11. Balkanci F, Ozmen MN. Case report: interruption of the inferior vena cava with anomalous intrahepatic continuation. $\mathrm{Br} J \mathrm{Ra}$ diol. 1993;66(785):457-9. doi: 10.1259/0007-1285-66-785-457. [PubMed 8319069].
12. Ghossain MA, Haddad SN, Aoun NJ, Atallah NG, Jebara VA, Moussally AS, et al. Portal and hemiazygos continuation of the inferior vena cava: report of an unusual case. Br J Radiol. 1988;61(724):333-4. doi: 10.1259/0007-1285-61-724-333. [PubMed:3370420].

13. Minniti S, Visentini S, Procacci C. Congenital anomalies of the venae cavae: embryological origin, imaging features and report of three new variants. Eur Radiol. 2002;12(8):2040-55. doi:10.1007/s00330-0011241-x. [PubMed: 12136323].

14. Kandpal H, Sharma R, Gamangatti S, Srivastava DN, Vashisht S. Imaging the inferior vena cava: a road less traveled. Radiographics. 2008;28(3):669-89. doi: 10.1148/rg.283075101. [PubMed: 18480478].

15. Mathews R, Smith PA, Fishman EK, Marshall FF. Anomalies of the inferior vena cava and renal veins: embryologic and surgical considerations. Urology. 1999;53(5):873-80. [PubMed: 10223477].

16. Yigit H, Yagmurlu B, Yigit N, Fitoz S, Kosar P. Low back pain as the initial symptom of inferior vena cava agenesis. AJNR Am J Neuroradiol. 2006;27(3):593-5. [PubMed:16551999].

17. Paksoy Y, Gormus N. Epidural venous plexus enlargements presenting with radiculopathy and back pain in patients with inferior vena cava obstruction or occlusion. Spine (Phila Pa 1976). 2004;29(21):241924. [PubMed: 15507805].

18. Jeong HJ, Seo IY, Rim JS. Chronic post-thrombotic obstruction of the inferior vena cava: both renal veins being the cause of painless gross hematuria from pelviureteral mucosal varices in normal functioning kidney. Yonsei Med J. 2003;44(6):1119-21. doi: 10.3349/ymj.2003.44.6.1119. [PubMed: 14703629].

19. Sonin AH, Mazer MI, Powers TA. Obstruction of the inferior vena cava: a multiple-modality demonstration of causes, manifestations, and collateral pathways. Radiographics. 1992;12(2):309-22. doi: 10.1148/radiographics.12.2.1561419. [PubMed: 1561419].

20. Dahan H, Arrive L, Monnier-Cholley L, Le Hir P, Zins M, Tubiana JM. Cavoportal collateral pathways in vena cava obstruction: imaging features. AJR Am J Roentgenol. 1998;171(5):1405-11. doi: 10.2214/ajr.171.5.9798887. [PubMed: 9798887].

21. Kapur S, Paik E, Rezaei A, Vu DN. Where there is blood, there is a way: unusual collateral vessels in superior and inferior vena cava obstruction. Radiographics. 2010;30(1):67-78. doi: 10.1148/rg.301095724. [PubMed: 20083586].

22. Albrechtsson $U$. The portal vein as collateral in inferior vena cava obstruction. Cardiovasc Radiol. 1979;2(2):107-10. [PubMed: 436129].

23. Wedig MP, Stark GB. [Upper gastrointestinal hemorrhage due to vena cava obstruction]. Z Kinderchir. 1990;45(6):379-82. doi:10.1055/s-20081042619. [PubMed: 2291343].

24. Maunoury V, Plane C, Ernst O, Seguy D, Quandalle P. [Absence of subrenal inferior vena cava revealed by rupture of duodenal varices in an adult]. Gastroenterol Clin Biol. 1994;18(10):889-91. [PubMed: 7875398].

25. Zhou H, Janssen D, Gunther E, Pfeifer U. Fatal bleeding from duodenal varices as a late complication of neonatal thrombosis of the inferior vena cava. Virchows Arch A Pathol Anat Histopathol. 1992;420(4):367-70. [PubMed: 1566566] 